\title{
Arginase 2 deficiency reduces hyperoxia-mediated retinal neurodegeneration through the regulation of polyamine metabolism
}

\author{
SP Narayanan ${ }^{\star, 1,2,3}, \mathrm{Z} \mathrm{Xu^{1,2 } , \mathrm { N } \mathrm { Putluri } ^ { 4 } , \text { A Sreekumar }}{ }^{4}$, T Lemtalsi $^{1,2}$, RW Caldwell ${ }^{5}$ and RB Caldwell ${ }^{1,2,3,6}$
}

Hyperoxia treatment has been known to induce neuronal and glial death in the developing central nervous system. Retinopathy of prematurity (ROP) is a devastating disease in premature infants and a major cause of childhood vision impairment. Studies indicate that, in addition to vascular injury, retinal neurons are also affected in ROP. Using an oxygen-induced retinopathy (OIR) mouse model for ROP, we have previously shown that deletion of the arginase 2 (A2) significantly reduced neuro-glial injury and improved retinal function. In the current study, we investigated the mechanism of A2 deficiency-mediated neuroprotection in the OIR retina. Hyperoxia treatment has been known to induce neuronal death in neonates. During the hyperoxia phase of OIR, a significant increase in the number of apoptotic cells was observed in the wild-type (WT) OIR retina compared with A2-deficient OIR. Mass spectrometric analysis showed alterations in polyamine metabolism in WT OIR retina. Further, increased expression level of spermine oxidase was observed in WT OIR retina, suggesting increased oxidation of polyamines in OIR retina. These changes were minimal in A2-deficient OIR retina. Treatment using the polyamine oxidase inhibitor, $\mathbf{N}, \mathbf{N}$-bis (2, 3-butadienyl)-1, 4-butanediamine dihydrochloride, significantly improved neuronal survival during OIR treatment. Our data suggest that retinal arginase is involved in the hyperoxia-induced neuronal degeneration in the OIR model, through the regulation of polyamine metabolism.

Cell Death and Disease (2014) 5, e1075; doi:10.1038/cddis.2014.23; published online 20 February 2014

Subject Category: Neuroscience

There has been increasing evidence that hyperoxia negatively affects neuronal development and maturation in neonates. It has been demonstrated that short exposures to nonphysiologic oxygen levels can trigger apoptotic neurodegeneration in the developing brain. ${ }^{1-3}$ Retinopathy of prematurity (ROP) is a potentially blinding eye disorder that primarily affects infants born prematurely. It is one of the most common causes of visual loss in childhood and can lead to lifelong vision impairment and blindness. Today, with advances in neonatal care, smaller and more premature infants are being saved and these infants are at a high risk for ROP. According to National Eye Institute, about 1100-1500 infants in the United States each year develop ROP that is severe enough to require medical treatment, and approximately 400-600 of these become legally blind from ROP.

From the clinical perspective, ROP is considered as a vascular disease and current treatments such as laser photocoagulation and cryotherapy target abnormal retinal angiogenesis. However, despite effective treatment of the vascular injury, many children suffer vision impairment, suggesting a disruption of neuronal development as well.
Considerable evidences support the concept that ROP is not only a vascular disorder, but is also associated with functional visual deficits in ROP patients ${ }^{4-8}$ as well as in animal models. ${ }^{9-12}$ Sennlaub et al. ${ }^{13}$ reported the first evidence for retinal neuronal apoptosis and degeneration in an animal model of ROP. Later, various studies including ours confirmed neuronal apoptosis and/or retinal degeneration in the ROP retina. ${ }^{14-18}$ However, the cellular mechanisms causing this neurodegeneration are still unknown. Oxygen treatment has been identified as a trigger of neuronal and glial apoptosis in the developing brain ${ }^{3,19,20}$ and adult retinal neurons. ${ }^{21-23}$ Hyperoxia-induced defects in retinal neural function have been reported in neonatal rats. ${ }^{24}$

Based on this evidence it is suggested that hyperoxiamediated neuronal death could be a major cause for visual dysfunction in ROP. However, the mechanisms of the neuronal injury are as yet unknown. Using the oxygeninduced retinopathy (OIR) mouse model of ROP, we have previously shown a significant increase in retinal cell death immediately following hyperoxia treatment. ${ }^{18}$ Our studies showed that deletion of arginase 2 (A2) significantly reduced

\footnotetext{
${ }^{1}$ Vision Discovery Institute, Georgia Regents University, Augusta, GA, USA; ${ }^{2}$ Vascular Biology Center, Georgia Regents University, Augusta, GA, USA; ${ }^{3}$ Department of Cellular Biology and Anatomy, Georgia Regents University, Augusta, GA, USA; ${ }^{4}$ Department of Molecular and Cellular Biology, Baylor College of Medicine, Houston, TX, USA; ${ }^{5}$ Department of Pharmacology and Toxicology, Georgia Regents University, Augusta, GA, USA and ${ }^{6}$ Charlie Norwood VA Medical Center, Augusta, GA, USA ${ }^{*}$ Corresponding author: SP Narayanan, Vascular Biology Center, Georgia Regents University, 1459 Laney Walker Boulevard, Augusta, GA 30912-2500, USA. Tel: + 1706721 7412; Fax: + 1706721 9799; E-mail: pnarayanan@ gru.edu

Keywords: arginase; polyamine; neurodegeneration; retinopathy of prematurity; hyperoxia

Abbreviations: ROP, retinopathy of prematurity; OIR, oxygen-induced retinopathy; RA, room air; WT, wild type; A2, arginase 2; SMO, spermine oxidase; TUNEL, terminal deoxynucleotidyl transferase (TdT) dUTP nick-end labeling; MDL 72527, N, N'-Bis (2, 3-butadienyl)-1, 4-butanediamine dihydrochloride; ONL, outer nuclear layer

Received 25.6.13; revised 27.12.13; accepted 07.1.14; Edited by P Salomoni
} 
neurodegeneration and improved retinal function. ${ }^{18}$ Arginase function is reported to be increased in various pathologies characterized by vascular dysfunction including diabetes ${ }^{25,26}$ hypertension ${ }^{27,28}$ and ischemia reperfusion injury. ${ }^{29,30}$ Overactive arginase can alter vascular function by decreasing the supply of L-arginine to nitric oxide synthase, which will decrease nitric oxide formation. However, arginase also can increase the formation of polyamines, which have been implicated in neurological disease conditions such as Alzheimer's disease ${ }^{31}$ and Parkinson's disease. ${ }^{32}$ Polyamine catabolism was found to be enhanced in a rat model of traumatic brain injury. ${ }^{33}$ Polyamines have been demonstrated to be involved in the pathogenesis of ischemic brain damage by various groups ${ }^{34-36}$ and implicated in exitotoxic neuronal death in retina. ${ }^{37}$ Amino aldehydes, acrolein and hydrogen peroxide are generated as byproducts during the oxidation of polyamines by polyamine oxidases and are toxic to neurons. ${ }^{38}$ Studies in vitro and in vivo have also demonstrated key roles for these reactive aldehydes in apoptotic and necrotic mechanisms leading to both neuronal and glial cell death. ${ }^{39-42}$ Polyamine regulated neurotoxicity is not well studied in retinopathy. However, elevated arginase activity and polyamine production have been linked to retinal ganglion cell death because of excessive activation of the excitotoxic NMDA receptors. ${ }^{37}$ In the present study, we investigated whether arginase/polyamine signaling mechanisms are associated with the neurodegeneration in ROP retina.

\section{Results}

Hyperoxia-induced cell death. Death of retinal cells during the hypoxic phase of OIR has been reported previously. ${ }^{13,17,18}$ It also has been shown that hyperoxia causes neuronal death in brain ${ }^{19}$ and retina. ${ }^{24}$ Hence, it is important to investigate retinal cell death in the OIR retina during the hyperoxic phase. In the present study, we analyzed hyperoxia-induced retinal apoptosis using terminal deoxynucleotidyl transferase (TdT) dUTP nick-end labeling (TUNEL) assay (Figure 1a). Compared with room air (RA) controls, no significant changes in the number of TUNEL-positive cells were observed in any OIR samples after $8 \mathrm{~h}$ of hyperoxia. However, after $24 \mathrm{~h}$ of hyperoxia there was a significant increase in the number of TUNEL-positive cells in the wild-type (WT) OIR retina compared with RA controls $(P<0.01)$. The hyperoxia-induced cell death was markedly reduced in the $A 2$ deficient $\left(A 2^{-/-}\right)$OIR retina compared with WT OIR at $24 \mathrm{~h}(P<0.01)$. The retinal cell death in the WT OIR retina continued as the hyperoxia treatment continued for $2-5$ days. During this period, the $A 2^{-1-}$ OIR retina showed significant neuroprotection as compared with the WT OIR retina. The number of TUNEL-positive cells in the $A 2^{-1-}$ OIR retina was significantly lower compared with the WT OIR at P9 (48 h of hyperoxia) as well (Figure $1 \mathrm{~b})$. The majority of TUNEL-positive cells are localized in the INL (Figure 1a); however, a few TUNEL-positive cells were observed in the outer nuclear (ONL) layer as well as in the ganglion cell layer (Supplementary Figure 1). Retinal cell death associated with normal development was observed in the RA controls and decreased as development continued.

Expression of arginase. Figure 2 shows the expression and localization of $\mathrm{A} 2$ on postnatal day 9 after 2 days of hyperoxia treatment in the OIR retina. We have previously reported that horizontal cells show elevated expression of A2 during the hypoxic phase of OIR. ${ }^{18}$ Our current analysis showed elevated levels of A2 in horizontal cells during the hyperoxia phase of OIR (Figures $2 a$ and $b$ ) as well. In addition, high levels of A2 expression were observed in the ONL of both RA and OIR retina (Figures $2 d$ and e). Expression of A2 was also seen in the region of the developing inner and outer segments (Figures $2 f$ and $g$ ). Studies have shown that development of photoreceptor inner and outer segments is not complete until postnatal day 15 in mouse retina. ${ }^{43}$ Western blot analysis showed no significant difference in the level of $A 2$ in the OIR retinas as compared with the RA control retinas (Figure $2 \mathrm{~h}$ ). This lack of an increase in total A2 protein levels despite the apparent
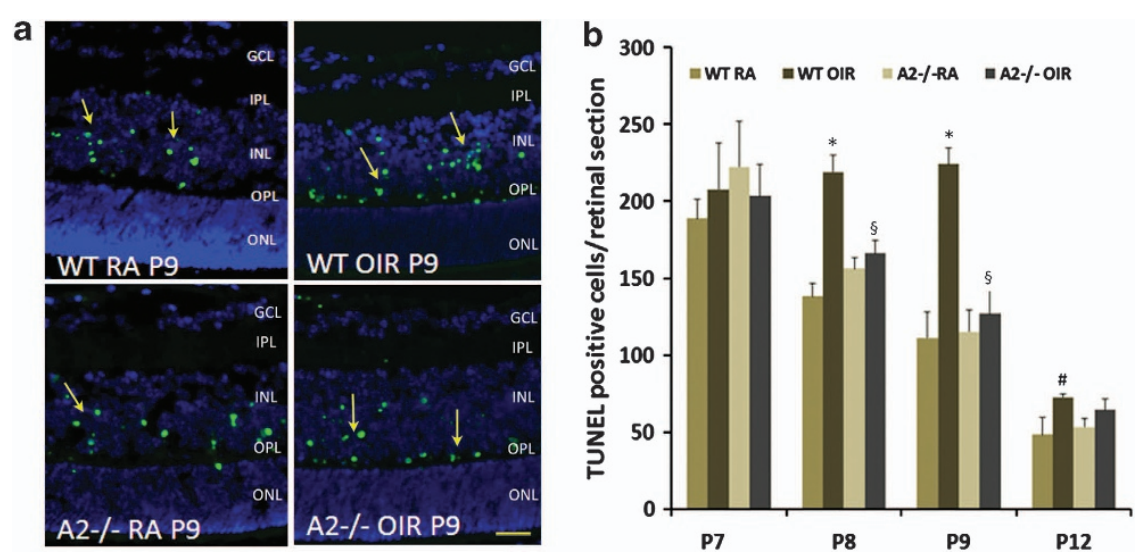

Figure 1 Hyperoxia-induced cell death in the OIR retina. (a) Cell death was studied using TUNEL assay on cryostat sections. Numerous apoptotic cells were present in the WT OIR retina. In the A2 ${ }^{-1}$ - OIR retina, fewer TUNEL-positive cells were observed compared with WT OIR retina. (b) Quantification of TUNEL-positive cells showed that the WT OIR retina had increased numbers of apoptotic cells at P8, P9 and P12 (1,2 and 5 days of hyperoxia, respectively). However, this increase was not observed in the A2 ${ }^{-1-}$ OIR retina. Data are presented as mean \pm s.d. "WT RA versus WT OIR $(P<0.01)$, "WT RA versus WT OIR $(P<0.05)$, ${ }^{\S} \mathrm{A} 2^{-l}$ - OIR versus WT OIR $(P<0.01), N$ varies from four to six. Scale bar $=50 \mu \mathrm{m}$. GCL, ganglion cell layer; INL, inner nuclear layer; IPL, inner plexiform layer; ONL, outer nuclear layer; OPL, outer plexiform layer 

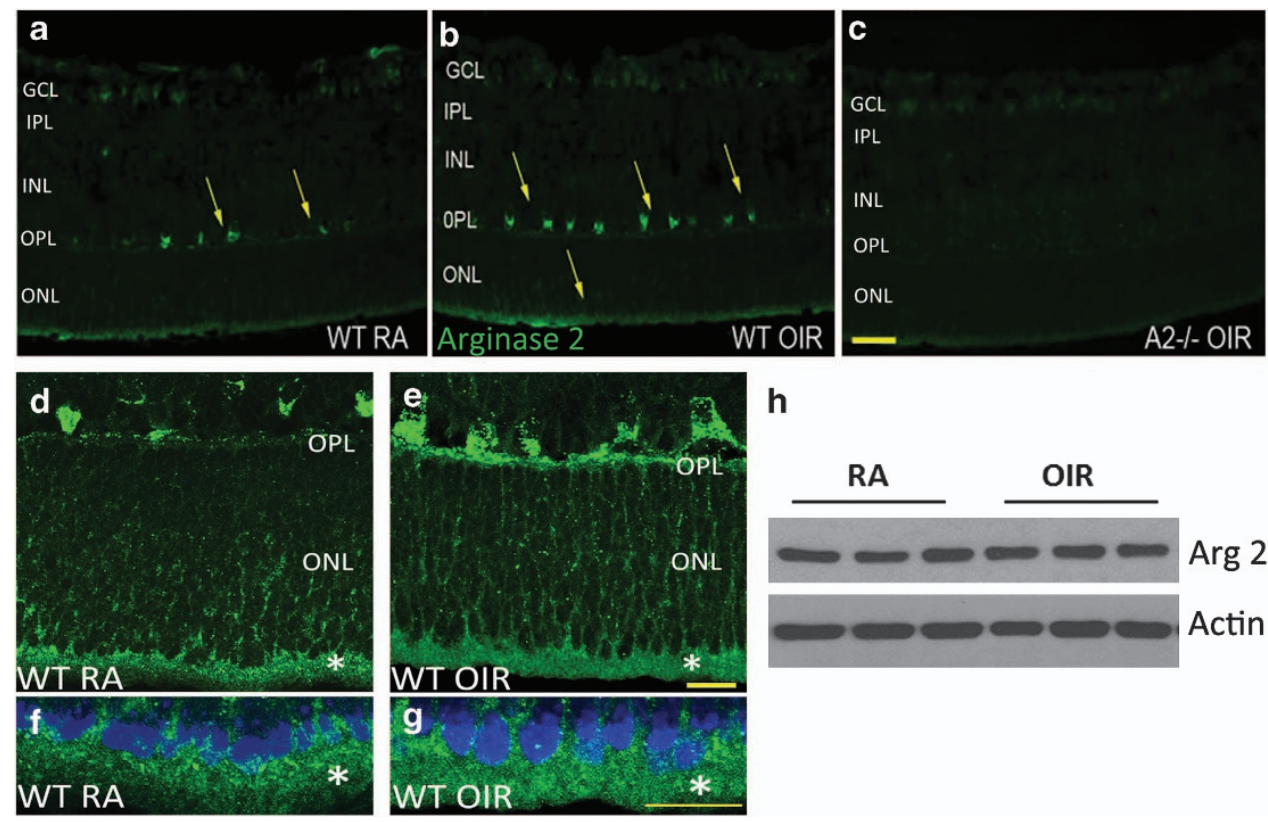

h

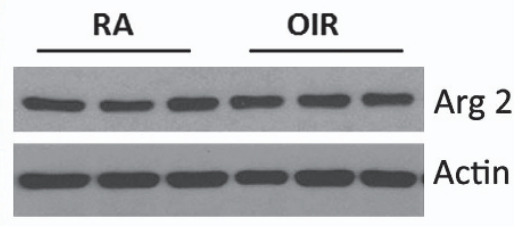

Figure 2 Expression of arginase 2 (A2) in the OIR retina. (a and $\mathbf{b}$ ) Immunostaining of retinal cryostat sections on postnatal day 9 showing immunoreactivity for A2 in horizontal cells and in the ONL in the WT RA and hyperoxia-treated WT OIR retina. (c) A2 ${ }^{-1}$ OIR retina used as negative control. Scale bar $=50 \mu \mathrm{m}$. (d and e) High-magnification confocal images of WT RA and WT OIR retina showing immunolocalization of A2 in the horizontal cells and outer nuclear layer. Scale bar $=20 \mu \mathrm{m}$. ( $\mathbf{f}$ and $\mathbf{g}$ ) High-magnification confocal images of A2 and DAPI showing A2 localization in the area of inner and outer segment development at P9. DAPI shows photoreceptor nuclei. Scale bar $=20 \mu \mathrm{m}$. (h) Western blot analysis showing the expression of A2 in RA and OIR retinas. GCL, ganglion cell layer; IPL, inner plexiform layer; INL, inner nuclear layer; ONL, outer nuclear layer; OPL, outer plexiform layer; *Area of inner and outer segment development

increase within the horizontal cells may be explained by degeneration of some horizontal cells as shown by quantitation of calbindin-positive INL cells (Figures $3 a$, b and c) and by TUNEL labeling of horizontal cells (Figure $3 d$ ). It should also be noted that the number of horizontal cells is very low relative to the number of photoreceptor cells, which express relatively high levels of $A 2$ in both RA controls and OIR retinas.

Polyamine oxidation in OIR retina. Increased arginase activity can lead to increased formation of polyamines. Polyamines and their oxidation products have been reported to be associated with neuronal death in various CNS pathologies. In the current study, we used mass spectrometry to analyze changes in the levels of polyamines and their acetylated intermediates in the OIR retina. Figure $4 \mathrm{a}$ shows a simplified flowchart of polyamine metabolism. As shown in Figure $4 \mathrm{~b}$, alterations in polyamine metabolism were evident in the OIR retina. A significant increase in spermidine along with a significant decrease in spermine was observed in the WT OIR retina. These alterations were not seen in the $A 2^{-1-}$ OIR retina. Spermidine levels in the $A 2^{-1-}$ OIR retina were significantly lower compared with WT OIR and similar to that of RA controls. Spermine was observed to be significantly increased in the $\mathrm{A} 2^{-1-}$ OIR retina compared with WT OIR. These results suggest the involvement of the backward conversion pathway of spermine to spermidine, by spermine oxidase (SMO) in the OIR retina. It has been well demonstrated that oxidation of spermine and spermidine
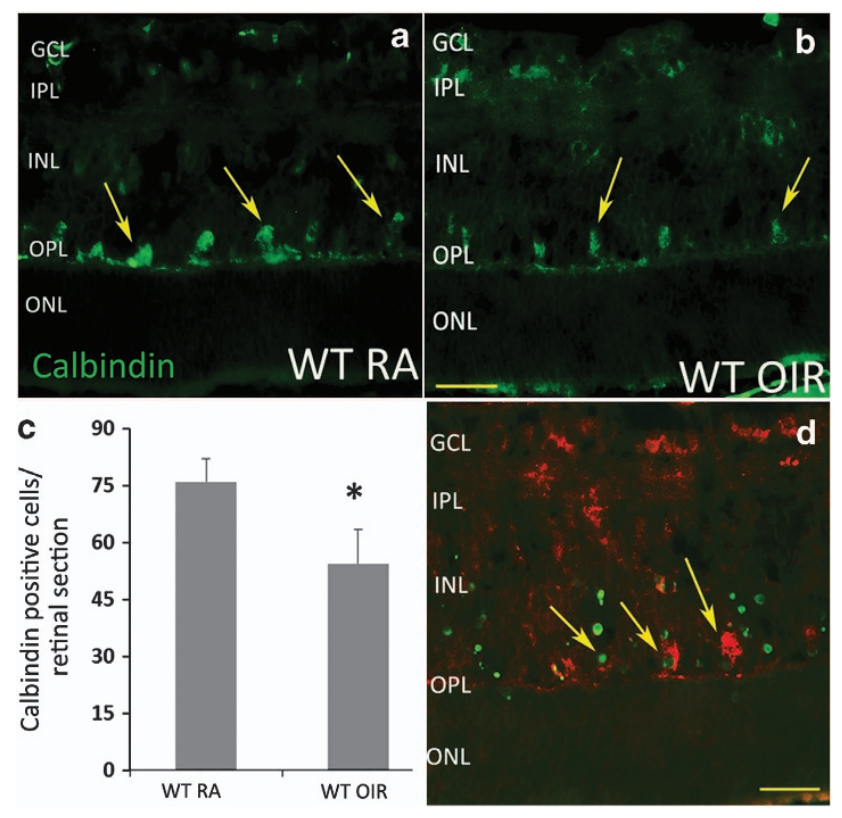

Figure 3 Analysis of horizontal cells in the WT OIR retina. ( $\mathbf{a}$ and $\mathbf{b}$ ) Calbindin immunostaining of horizontal cells on RA and OIR sections. Scale bar $=50 \mu \mathrm{m}$. (c) Quantification showing a significant decrease in the number of calbindin-positive horizontal cells in WT OIR retina compared with RA controls. ${ }^{*} P<0.05, N=5$. (d) Colocalization of TUNEL-positive cells with calbindin-positive horizontal cells in WT OIR retina. Scale bar $=50 \mu \mathrm{m}$. GCL, ganglion cell layer; INL, inner nuclear layer; IPL, inner plexiform layer; ONL, outer nuclear layer; OPL, outer plexiform layer 
a

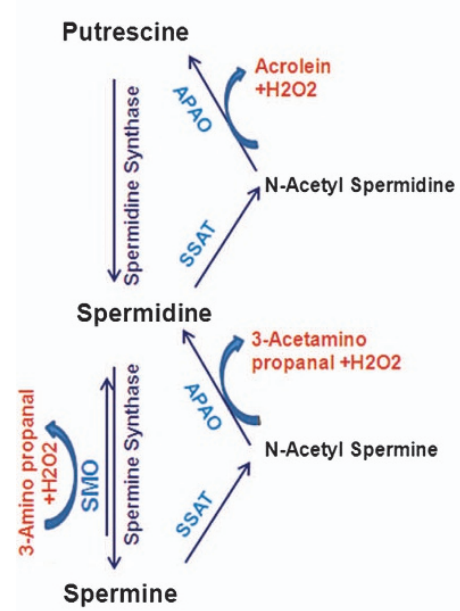

b
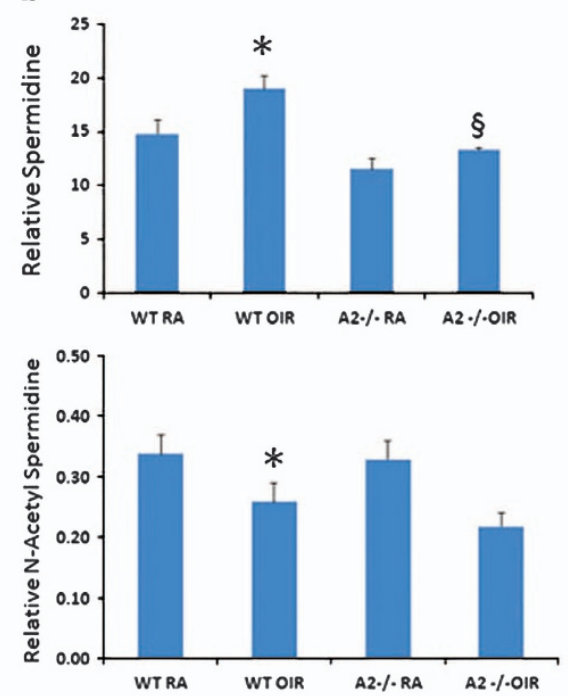
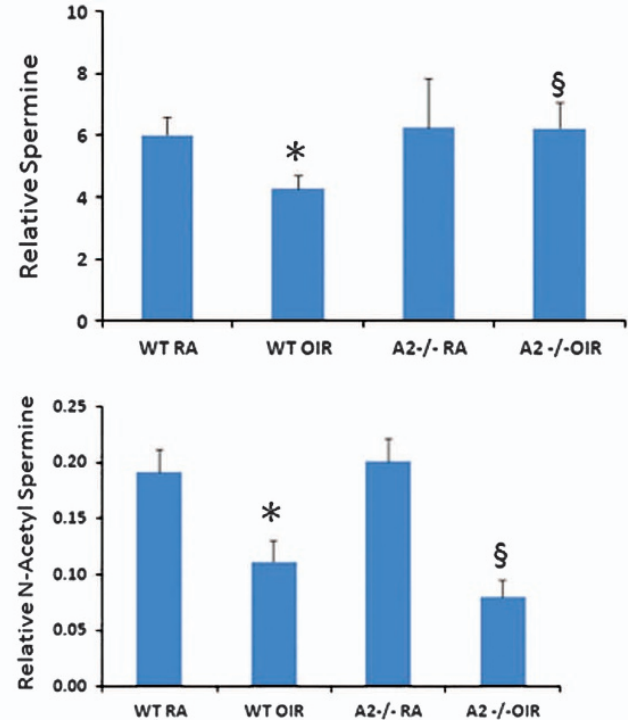

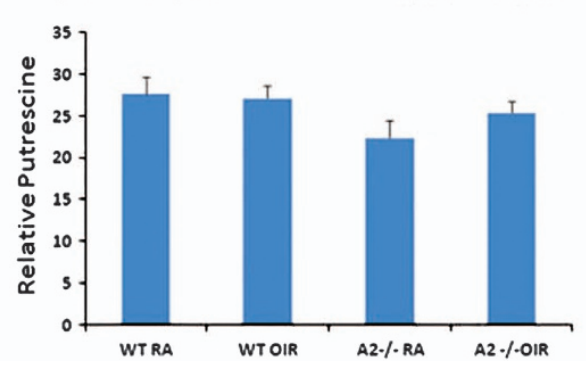

Figure 4 Analysis of polyamines in OIR retina. (a) Simplified flow chart showing the polyamine interconversion pathway. SMO, spermine oxidase; APAO, acetyl polyamine oxidase; SSAT, spermine spermidine acetyl transferase. Modified from Narayanan et al. ${ }^{44}$ (b) Using mass spectrometry, levels of spermine, spermidine, putrescine, $\mathrm{N}$-acetyl spermine and $\mathrm{N}$-acetyl spermidine were analyzed in the retinal samples collected on $\mathrm{P} 9$ (2 days of hyperoxia) and their relative levels are normalized to internal standards used. Changes in polyamine metabolism are observed in WT OIR retina. Data presented as mean \pm S.E.M. ${ }^{*}$ WT RA versus WT OIR $(P<0.05),{ }^{\circledR}$ A2 ${ }^{-1-}$ OIR versus WT OIR $(P<0.05), N$ varies from 10 to 12

generates reactive aldehydes such as 3-amino propanal and acrolein, and $\mathrm{H}_{2} \mathrm{O}_{2}$ as by products. ${ }^{38,45}$ As seen in Figure $4 b$, putrescine levels were not significantly changed in any OIR retina relative to normoxia controls. Levels of acetylated spermine and spermidine were reduced in both OIR groups. $\mathrm{N}$-Acetyl spermine, the intermediate in the oxidation of spermine to spermidine, was significantly increased in WT OIR compared with $A 2^{-I-}$ OIR retina.

Expression and activation of SMO. Polyamine oxidases are enzymes involved in the backward oxidation of spermine and spermidine to spermidine and putrescine, respectively. $\mathrm{SMO}$ is the polyamine oxidase that converts spermine to spermidine. We further investigated the expression of SMO in the OIR retina. Significant increases in SMO expression was observed in the WT retina at all stages of OIR studied (Figures $5 \mathrm{a}$ and b). Increased expression of SMO was observed in WT OIR in comparison with WT RA as early as $\mathrm{P} 8$ and continued through $\mathrm{P} 12$. In the $\mathrm{A} 2^{-/-}$OIR retina, levels of SMO were significantly reduced compared with WT OIR and levels were similar to WT RA controls. However, in the $\mathrm{A} 2^{-1-} \mathrm{RA}$ controls, levels of SMO were elevated as compared with WT controls. Immunolocalization studies using confocal imaging showed that SMO expression is distributed in the ganglion cell layer, INL, OPL, ONL and RPE cells (Figures $5 c-f$ ). Consistent with western blot data, the $\mathrm{A} 2^{-1-}$ OIR retina showed reduced expression of SMO. Higher magnification images showed increased SMO immunoreactivity in the OPL and ONL of the WT OIR retina (Figures 5g-j). As photoreceptor inner and outer segments are not well differentiated at this time, it was not possible to localize SMO to specific photoreceptor compartments. SMO expression was also prominent in the RPE cells of the WT OIR and $A 2^{-1-} \mathrm{RA}$ retinas compared with the other groups (Figures $5 k-n$ ). Reactive aldehydes such as 3-amino propanal are generated along with $\mathrm{H}_{2} \mathrm{O}_{2}$ as byproducts of polyamine oxidation. These can increase oxidative stress and are cytotoxic leading to neuronal death. Formation of $\mathrm{H}_{2} \mathrm{O}_{2}$ in the OIR retinas was studied using Amplex Red assay. As shown in Figures $50, \mathrm{H}_{2} \mathrm{O}_{2}$ release was significantly increased in WT OIR retina. In $\mathrm{A} 2^{-1-}$ OIR retina, $\mathrm{H}_{2} \mathrm{O}_{2}$ release was significantly reduced relative to WT OIR retina. These results suggest that oxidation of spermine is reduced in the $A 2^{-1-}$ OIR retina.

Apoptotic signaling pathways. We further studied the signaling pathway involved in the polyamine oxidationmediated neuronal death in the retina. Increased phosphorylation of JNK/SAPK was observed in WT OIR retina compared with normoxic controls. An elevated expression 

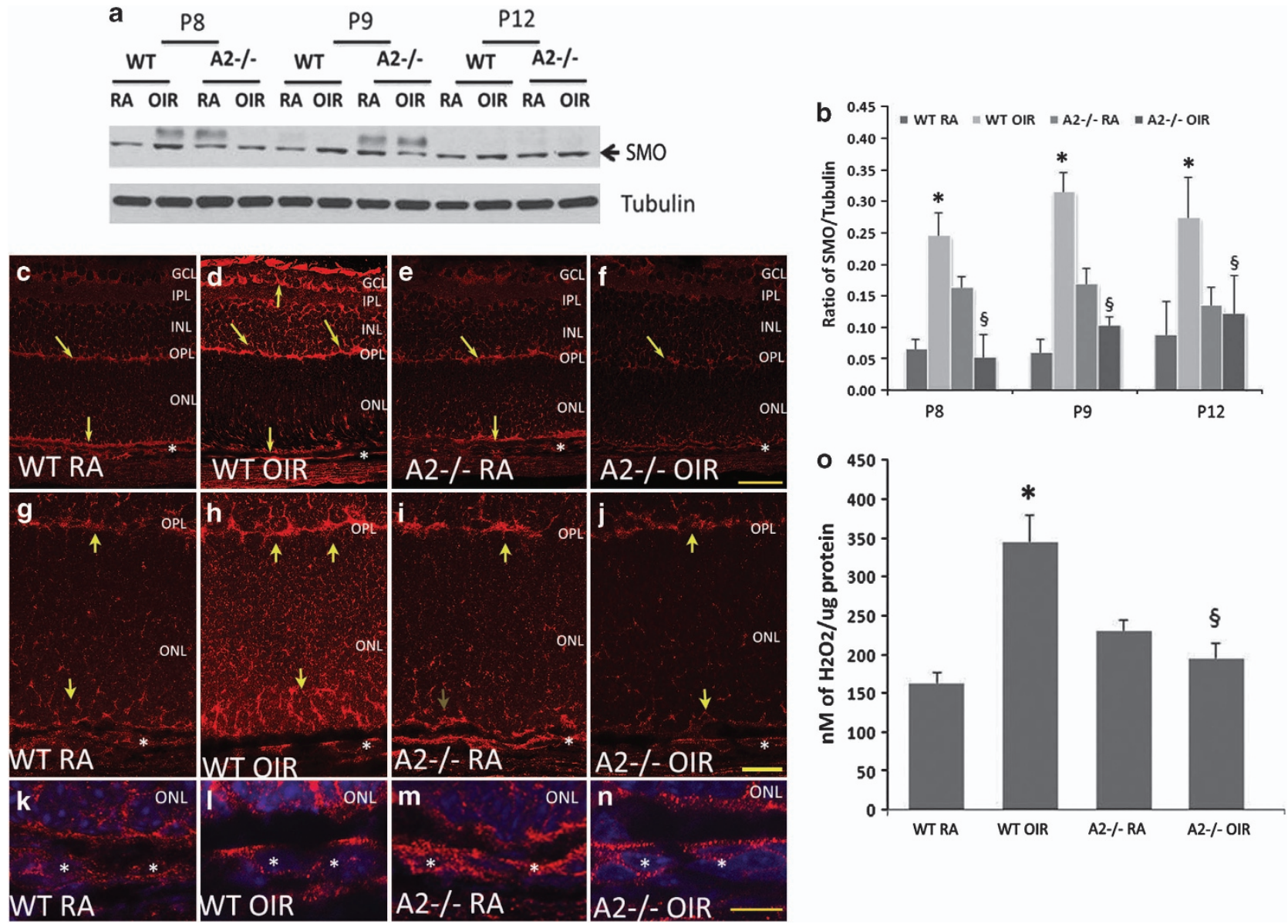

Figure 5 Increased polyamine oxidation in the OIR retina. (a) Western blot analysis showing increased expression of spermine oxidase (SMO) in WT OIR retina during different stages of hyperoxia. SMO expression is significantly reduced in $\mathrm{A} 2^{-1-}$ OIR retina and is comparable to RA controls at all stages of hyperoxia studied. (b) Quantification of SMO expression in RA and OIR samples using ImageJ software. Data presented as mean \pm S.D. ${ }^{*}$ WT RA versus WT OIR $(P<0.01),{ }^{\S}$ A2 ${ }^{-l}-{ }^{\circ}$ OIR versus WT OIR $(P<0.01)$, N varies from 4 to 6 . (c-n) Confocal images of SMO immunolocalization on retinal cryostat sections on postnatal day 9. Top panel (c-f) shows SMO expression in various retinal layers. Arrows represent areas of high expression. Scale bar $=50 \mu \mathrm{m}$. Middle panel ( $\mathbf{g}-\mathbf{j})$ shows increased expression of SMO in the OPL and ONL in WT OIR retina. Scale bar $=20 \mu \mathrm{m}$. High-magnification confocal images of SMO and DAPI in the bottom panel $(\mathbf{k}-\mathbf{n})$ show SMO expression in the ONL and RPE cells. GCL, ganglion cell layer; INL, inner nuclear layer; IPL, inner plexiform layer; ONL, outer nuclear layer; OPL, outer plexiform layer, ${ }^{*} \mathrm{RPE}$. Scale bar $=10 \mu \mathrm{m}$. (o) Analysis of hydrogen peroxide formation in the OIR retina using Amplex red showing increased $\mathrm{H}_{2} \mathrm{O}_{2}$ in WT OIR retina compared with A2 ${ }^{-l-}$ OIR and room air controls. Data presented as mean \pm S.D. ${ }^{*}$ WT RA versus WT OIR $(P<0.01),{ }^{\circledR}$ A2 ${ }^{-l-}$ OIR versus WT OIR $(P<0.01), N=5$

level of FASL was also observed in the WT OIR retina compared with RA controls, demonstrating the involvement of the death receptor pathway. These changes were not observed in $\mathrm{A} 2^{-1-}$ OIR retina (Figures $6 \mathrm{a}$ and $\mathrm{b}$ ). In addition, markedly increased levels of BID, cleaved BID and cytochrome $c$ were observed in WT OIR retina as compared with the RA controls. These results indicate that the mitochondrial permeability pathway is also involved. In the $A 2^{-1-}$ OIR retina, the expression of BID, cleaved BID and cytochrome $c$ were not elevated, and instead were significantly reduced compared with the WT OIR (Figures $6 a$ and b), consistent with decreased neuronal death in the $\mathrm{A} 2^{-/-}$OIR retina.

Inhibition of polyamine oxidase. In order to determine the impact of inhibiting polyamine oxidation on neuronal cell death, mice were treated with polyamine oxidase inhibitor, MDL72527, on P7 and P8, and retinal cell death was examined on P9. WT RA animals were used as controls to see whether MDL 72527 ( $N, N^{\prime}$-bis (2, 3-butadienyl)-1, 4-butanediamine dihydrochloride) had any effect on developmental apoptosis. WT OIR mice treated with MDL 72527 for 2 days showed significantly reduced apoptosis in the retina compared with vehicle-treated controls (Figures $7 \mathrm{a}$ and $b$ ). These results further confirm the role of polyamine oxidation in neuronal death in the OIR retina. RA controls treated with vehicle or MDL 72527 did not show any significant change in numbers of TUNEL-positive cells. Analysis of $\mathrm{H}_{2} \mathrm{O}_{2}$ formation using amplex red assay showed reduced levels of $\mathrm{H}_{2} \mathrm{O}_{2}$ in the MDL 72527-treated OIR retina compared with vehicle-treated controls (Figure 7c). These results demonstrate that inhibiting polyamine oxidation during hyperoxia treatment reduces neuronal death in the retina.

Studies of apoptotic signaling mechanisms showed that levels of phosphorylated JNK/SAPK as well as total 
a

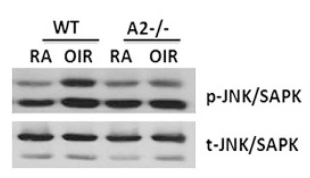

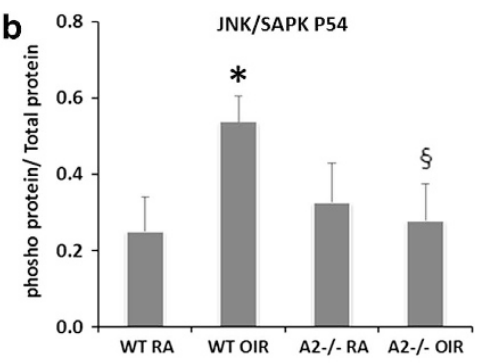

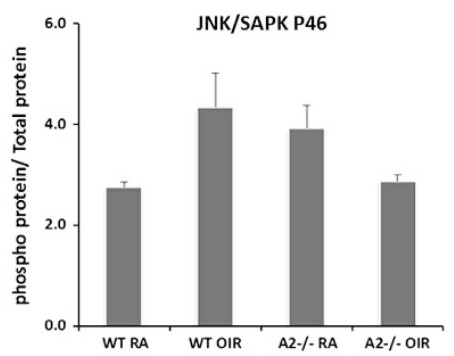

C
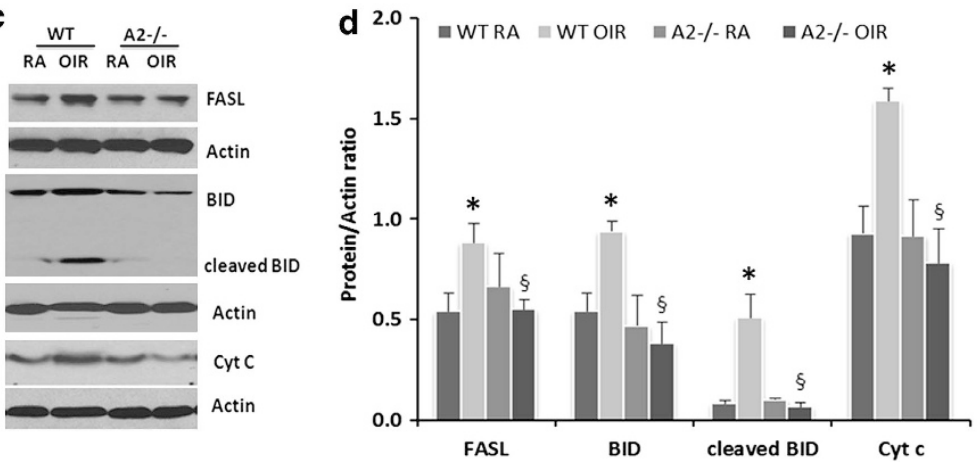

Figure 6 Signaling mechanisms of hyperoxia-mediated neuronal death in OIR retina. (a) Western blot analysis showing the phosphorylation levels of JNK/SAPK P54 and JNK/SAPK P46. (b) Quantification of phosphorylation changes in JNK/SAPK P54 and JNK/SAPK 46. (c) Western blot analysis showing the expression of FASL, BID, cleaved BID and cytochrome c in WT OIR retina as compared with $A 2^{-1-}$ OIR retina and RA controls. (d) Quantification of changes in these signaling molecules in WT RA, WT OIR, A2 ${ }^{-1-}$ $\mathrm{RA}$ and A2 ${ }^{-1-}$ OIR retinas. Data are presented as mean \pm S.D. ${ }^{*}$ WT RA versus WT OIR $(P<0.01),{ }^{\S}$ A2 ${ }^{-1-}$ OIR versus WT OIR $(P<0.01), N$ varies from four to six
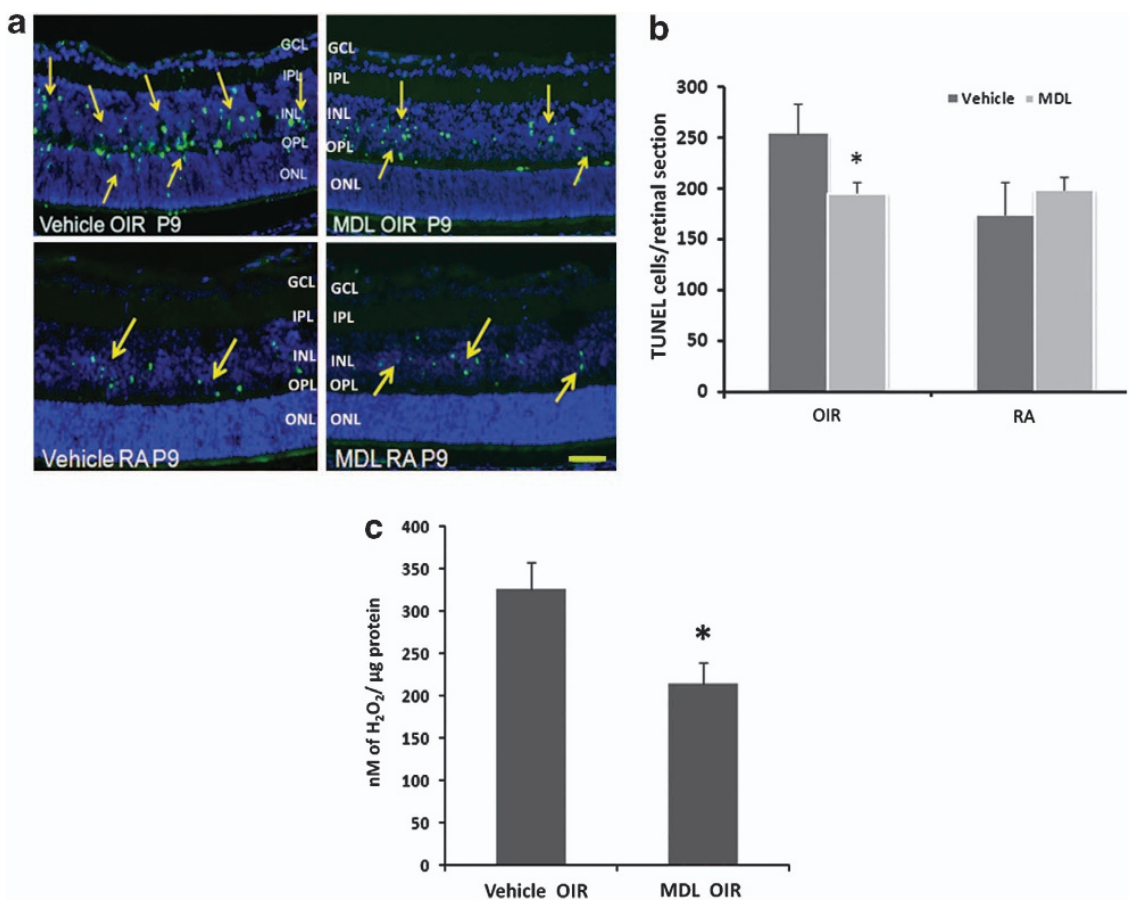

Figure 7 Impact of polyamine oxidase inhibition in the OIR retina. (a) TUNEL assay performed on retinal sections from RA and OIR mice treated with vehicle or MDL 72527 from P7 to P9. (b) Quantification of the number of TUNEL-positive cells per retinal section in RA and OIR retina treated with vehicle or the polyamine oxidase inhibitor MDL 72527, showing significant neuronal survival in MDL 72527-treated OIR retina. (c) Measurement of $\mathrm{H}_{2} \mathrm{O}_{2}$ formation using Amplex Red assay in the RA and OIR retina treated with vehicle or MDL 72527. Data presented as mean \pm S.D. ${ }^{*}$ Vehicle OIR versus MDL 72527 OIR $(P<0.01), N$ varies from four to six. Scale bar $=50 \mu \mathrm{m}$. GCL, ganglion cell layer; INL, inner nuclear layer; IPL, inner plexiform layer; ONL, outer nuclear layer; OPL, outer plexiform layer

JNK/SAPK were significantly reduced in MDL72527-treated OIR retina compared with vehicle-treated OIR group. Significant reductions in the expression of FASL and cytochrome $c$ were also observed in MDL 72527-treated OIR retina (Figures $8 a$ and $b$ ). These results demonstrate that inhibiting polyamine oxidation can block hyperoxia-induced 

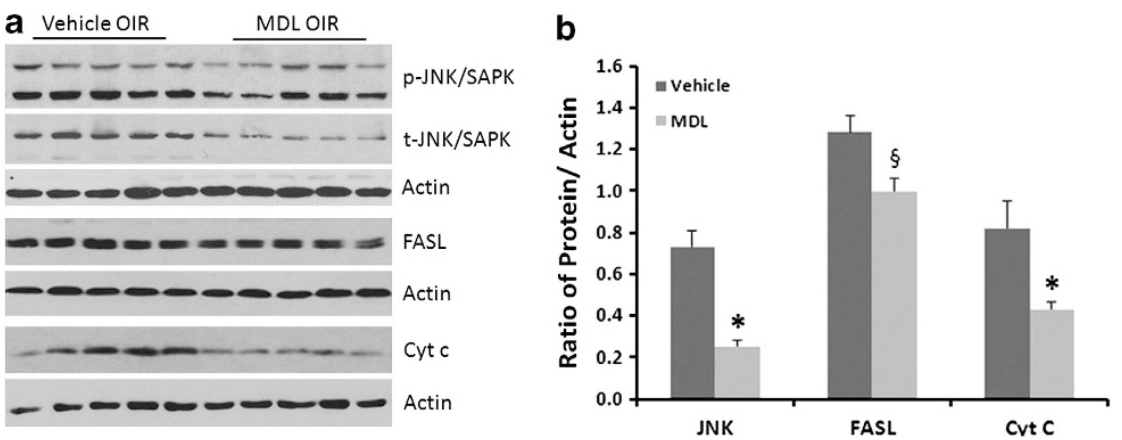

Figure 8 Effect of MDL 72527 treatment on apoptotic signaling in the OIR retina. (a) Western blot analysis showing changes in p-JNK/SAPK, t-JNK/SAPK FASL and cytochrome $c$ with MDL 72527 treatments. (b) Quantification of changes in apoptotic signaling molecules in response to MDL 72527 treatment in the OIR retina. Data presented as mean \pm S.D. *Vehicle OIR versus MDL 72527 OIR $(P<0.01)$, ${ }^{\S}$ Vehicle OIR versus MDL 72527 OIR $(P<0.05), N=5$

activation of FASL and mitochondrial permeability-mediated cell death pathways in the OIR retina.

\section{Discussion}

The present study provides the first specific evidence for hyperoxia-induced neuronal death during OIR and polyamine oxidation as a novel mediator of neurodegeneration during ROP. Although retinal neovascularization is the most recognized characteristic of ROP, the disease results in neuronal dysfunction as well. ${ }^{6,7,9,46,47}$ However, the cellular mechanisms are still not well understood. We have previously shown that deletion of $\mathrm{A} 2$ significantly reduces retinal degeneration and improves retinal function following hyperoxia treatment in the mouse model of OIR. ${ }^{18}$ In the present study, we are demonstrating the mechanism of this neuroprotective effect. Neuronal degeneration during OIR has been studied previously by several groups. ${ }^{14-18}$ However, these previous studies have focused on the hypoxia phase of OIR (P12-P17). In the current study, we report significant neuronal death during the hyperoxia phase. To the best of our knowledge, this is the first report of hyperoxia-induced retinal neuronal apoptosis during OIR. In our previous study, we have shown that TUNEL-positive cells in the WT OIR retina mainly included INL neurons such as amacrine cells and bipolar cells, but also included some ganglion cells, photoreceptors and Muller cells. In the present work, we have not characterized the TUNEL-positive cells. However, it is evident that they are localized mainly in the inner retina. This suggests that various retinal neurons could be undergoing hyperoxiainduced cell death. The significant reduction in numbers of TUNEL-positive cells in hyperoxia-treated $\mathrm{A} 2^{-1-}$ mice demonstrates a role for $\mathrm{A} 2$ in hyperoxia-induced retinal neurodegeneration.

We have shown that $A 2$ is strongly expressed in retinal horizontal cells. ${ }^{18}$ Immunoreactivity for A2 appears higher in horizontal cells of the hyperoxia-treated OIR retina. However, western blot analysis showed no change in the total levels of A2 protein in OIR retina compared with RA controls. We believe that this could be due to the degeneration of some of the horizontal cells under hyperoxia conditions. Lachapelle et al. $^{47}$ have reported degeneration of horizontal cells in newborn rats under hyperoxia. It is interesting that while the major localization of A2 is in horizontal cells, its deficiency is neuroprotective for retinal neurons in the INL. This could be due to the release of neurotoxic factors from degenerating horizontal cells and/or alterations in cell-cell interactions resulting from the degeneration of A2-expressing cells.

It has long been known that arginase is an important regulator of polyamine metabolism. ${ }^{48}$ Alterations in polyamine metabolism are linked with neurodegeneration in various pathologies. Increases in levels of spermidine relative to spermine have been reported in neurodegenerative conditions such as Alzheimer's disease ${ }^{31}$ and Parkinson's disease. ${ }^{32}$ Polyamines are also involved in the pathogenesis of ischemic brain damage. ${ }^{34-36}$ Polyamine-regulated neurotoxicity is not well studied in retinopathy. However, elevated arginase activity and polyamine production have been linked to retinal ganglion cell death because of excessive activation of the excitotoxic NMDA receptors. ${ }^{37}$ In the present study, we have demonstrated the involvement of polyamine oxidation in hyperoxia-mediated neuronal death. Increased levels of spermidine and decreased levels of spermine in the WT OIR retina (Figure 4b) suggest backward conversion of spermine to spermidine catalyzed by SMO. This was further confirmed by data showing increased expression of SMO in the WT OIR retina. SMO is a key enzyme in polyamine metabolism. ${ }^{38}$ Its function is important for maintaining polyamine homeostasis in mammalian cells. SMO catalyzes the oxidation of spermine to spermidine. During polyamine catabolism, amino aldehydes, acrolein and hydrogen peroxide are generated as byproducts of polyamine oxidase activity and these are shown to be toxic during ischemic brain injury. ${ }^{38}$

The mechanism by which Arginase regulates SMO expression/activity in the OIR retina is not known. One possible explanation is that hyperoxia-induced increases in arginase function tend to increase ornithine and polyamine levels, which in turn activate SMO to maintain the balance in intracellular polyamines. Under normoxia conditions in the $\mathrm{A} 2^{-1-}$ retina, polyamine synthesis is relatively low (as seen by reduced spermidine and putrescine) because of the arginase deficiency and the level of SMO is higher. This could be a compensatory mechanism to maintain the supply of intracellular spermidine. When the $\mathrm{A} 2^{-1-}$ retina is exposed to hyperoxia, SMO expression/activity is decreased in order to reduce spermine oxidation and thus preserve the intracellular pool of spermine and spermidine. Consistent with the suggested backward conversion of spermine to spermidine 
in the WT OIR retina, levels of both acetylated polyamines ( $N$-acetyl spermine and $N$-acetyl spermidine) were significantly reduced in the WT OIR retina as compared with the WT RA controls. In the $\mathrm{A} 2^{-1-}$ OIR retina, $\mathrm{N}$-acetyl spermine was significantly reduced compared with the WT OIR retina. This could be due either to decreased acetylation of spermine or increased oxidation of $\mathrm{N}$-acetylated spermine. As the level of spermidine level is also significantly lower in $A 2^{-1-}$ OIR retina as compared with the WT OIR retina, it is possible that acetylation of spermine by the enzyme, SSAT (spermine spermidine acyl transferase) is reduced in the absence of A2. In the present study, we have not investigated SSAT expression or activity. However, the amount of $\mathrm{N}$-acetylated spermidine was similar in both OIR groups. We plan to determine the role of SSAT in OIR in our future studies.

Considering that the peak of apoptosis occurred at P9 after $48 \mathrm{~h}$ hyperoxia, we studied polyamine levels at P9. It is very likely that polyamines and their metabolites are also altered earlier. These alterations in polyamine metabolism are further confirmed by elevated expression of SMO at all stages of hyperoxia studied. Localization studies showed that SMO is expressed by cells in the inner and outer retina.

Studies in developing brain have shown the critical involvement of Fas signaling in the pathogenesis of hyperoxia-mediated injury ${ }^{19}$ as well as in other types of neuronal injuries. ${ }^{19,49,50}$ Recently, Sifringer et al. ${ }^{51}$ showed that hyperoxia-mediated neurodegeneration in the developing brain is supported by intrinsic apoptosis as well. Our studies show that phosphorylation of $p$-JNK/SAPK and expression of FASL was increased in the OIR retina. In addition, expression of BID (and cleaved BID) and cytochrome $c$ were also elevated in the OIR retina. During Fas signaling, full-length BID, localized in cytoplasm, is cleaved by caspase 8 and cleaved BID (tBID) translocate to the mitochondrial outer membrane and triggers caspase 9-mediated apoptotic signaling and cytochrome $c$ release. ${ }^{52,53}$ Increased cytochrome $c$ release supports the involvement of mitochondrial membrane permeability-mediated apoptosis in the OIR retina. In our earlier studies, we have shown the involvement of the intrinsic pathway in neuronal death during the hypoxic phase of OIR. ${ }^{18}$ Expression of the apoptotic markers studied was reduced in $\mathrm{A} 2^{-/-}$OIR retina, suggesting that deficiency of $\mathrm{A} 2$ reduces apoptotic signals during OIR. Various studies in brain have shown the involvement of both the intrinsic pathway ${ }^{51,54}$ and Fas signaling pathways ${ }^{19,50}$ in hyperoxia-induced neuronal death. Acrolein has been shown to exert direct mitochondrial toxicity, ${ }^{55}$ whereas aminoaldehydes like 3-amino propanal act at the premitochondrial phase of apoptosis via promoting lysosomal leakage or lysis. ${ }^{56,57}$

In the current study, treatment of OIR retina using PAO inhibitor significantly reduced retinal apoptosis and $\mathrm{H}_{2} \mathrm{O}_{2}$ formation compared with vehicle-treated group. MDL 72527 is a competitive irreversible inhibitor of SMO and APAO, and is widely used to inhibit polyamine oxidase activity. ${ }^{58}$ Studies have shown that inhibition of polyamine oxidases using MDL 72527 significantly reduced brain edema, ischemic injury volume and polyamine levels in a rat model of cerebral ischemia. ${ }^{45,59}$ In another study, Dogan et al. have shown that blockade of the polyamine interconversion pathway using MDL 72527 can be neuroprotective after traumatic brain injury. ${ }^{59}$ The reactive aldehydes and $\mathrm{H}_{2} \mathrm{O}_{2}$ released during polyamine oxidation can damage RNA, DNA, proteins and membranes. This oxidative damage can lead to apoptosis of cells and the heritable damage to DNA may result in transformation, carcinogenesis and metastatic disease. ${ }^{45}$ In our system MDL 72527 must be blocking the oxidation of spermine and/or spermidine, and thus reducing the formation of $\mathrm{H}_{2} \mathrm{O}_{2}$ and other toxic aldehydes, thereby reducing oxidative stress to OIR retina.

To the best of our knowledge, this is the first report elucidating hyperoxia-induced neuronal damage in a model of OIR. We have demonstrated a crucial role of the arginase/ polyamine pathway in the OIR retina and oxidation of polyamines as one of the major mechanisms associated with neuronal injury in the OIR retina. Considering the need for new therapies for patients suffering from vision problems associated with ROP, our findings are highly relevant from a clinical perspective. Our data suggest that inhibition of arginase/ polyamine signaling can be considered as a therapeutic target to limit neuronal dysfunction in ROP patients.

\section{Materials and Methods}

Antibodies. Antibodies were purchased from SantaCruz Biotechnology (Dallas, TX, USA) (A2, Polyamine Oxidase), Sigma (St. Louis, MO, USA) (Calbindin, Tubulin) and Cell Signaling Technology (Boston, MA, USA) (FASL, P-JNK/SAPK, total JNK/SAPK, BID and cytochrome c).

Animal model of OIR. This study was carried out in strict accordance with the recommendations in the Guide for the Care and Use of Laboratory Animals of the National Institutes of Health. Experiments were approved by the Institutional Animal Care and Use Committee of the Georgia Regents University. Mice deficient in arginase $2\left(\mathrm{~A}^{-l-}\right)$ and WT controls were used in these studies. $\mathrm{A} 2^{-1-}$ mice developed by Shi et $a l^{60}$ were provided by Dr. Cederbaum (University of California, Los Angeles, California, USA) with the permission of Dr. O'Brien. The mouse model for OIR was generated as described previously. ${ }^{18}$ Briefly, on P7 newborn mice are placed with their dam in $70 \%$ oxygen (hyperoxia) for 5 days and then returned to RA on P12. Mice were killed at various times during the hyperoxia treatment and their retinas were collected and analyzed as described below.

TUNEL assay. Cell death was studied using TUNEL assay on cryosections (Flourescein Insitu Cell death detection kit, Millipore, Billerica, MA, USA). Quantification of TUNEL-positive cells in retinal cryosections from optic disc to periphery was performed manually. A minimum of three sections (20 $\mu \mathrm{m}$ apart) per animal were used.

Immunofluorescence. This was performed as described previously according to Narayanan et al. ${ }^{18}$ Briefly, eyes were enucleated, fixed in $4 \%$ paraformaldehyde (overnight, $4^{\circ} \mathrm{C}$ ), washed in PBS and cryoprotected overnight in $30 \%$ sucrose. Cryostat sections $(10 \mu \mathrm{m})$ were permeabilized in $1 \%$ Triton X-100 $(20 \mathrm{~min})$ and blocked in 10\% normal goat serum containing $1 \%$ BSA $(1 \mathrm{~h})$. Sections were then incubated overnight in primary antibodies $\left(4^{\circ} \mathrm{C}\right)$. On day two, they were incubated $(1 \mathrm{~h})$ in Flourescein or Texas red-conjugated secondary antibodies (Life Technologies, Grand Island, NY, USA), washed in PBS and mounted with Vectashield (Vector Laboratories, Burlingame, CA, USA).

Microscopy. Imaging of retinal cryostat sections was performed using either confocal microscope (Zeiss LSM 510 META) or a Zeiss Axioplan Imager Microscope (Carl Zeiss, Thornwood, NY, USA).

Quantification of horizontal cells. Calbindin-positive INL cells were quantified on retinal cryostat sections manually from optic disc to periphery. A minimum of three sections ( $20 \mu \mathrm{m}$ apart) per animal were used.

Western blotting. Retinal homogenates were prepared using RIPA buffer (Millipore) supplemented with protease and phosphatase inhibitors (Roche Applied Sciences, Indianapolis, IN, USA). Proteins were separated on SDS-PAGE and 
transferred onto nitrocellulose membrane (Millipore), blocked in 5\% milk or $3 \%$ BSA in TBST (Tris-buffered saline containing $0.5 \%$ Tween 20). The membrane was incubated overnight $\left(4^{\circ} \mathrm{C}\right)$ in primary antibodies diluted in the blocking solution, washed and incubated in respective secondary antibodies (HRP conjugated, GE Healthcare, Pittsburgh, PA, USA) for $1 \mathrm{~h}$ at room temperature and developed using Enhanced Chemiluminescence (ECL, GE Healthcare) or Westdura Detection (Thermo Scientific, Rockford, IL, USA).

Liquid chromatography/mass spectrometry: sample preparation. Retinal tissues were homogenized in ice-cold water/methanol (1:4) mixture containing equimolar mixture of standard compounds ([15N]-Tryptophan, [D4] Thymine [15N] Arginine). The homogenate was then subjected to extraction with sequential use of aqueous and organic solvents in the ratio $1: 4: 3: 1$ (water/methanol/chloroform/water). The resulting extracts were de-proteinized using a $3-\mathrm{kDa}$ molecular filter (Amicon Ultracel $-3 \mathrm{~K}$ Membrane, Millipore Corporation, Billerica, MA, USA) and the filtrate containing metabolites were dried under vacuum (Genevac EZ-2 plus , Genevac, Gardiner, NY, USA). Before mass spectrometry analysis, the dried extract was resuspended in identical volume of injection solvent composed of water/methanol $(50: 50)$ with $0.2 \%$ acetic acid and subjected to liquid chromatography mass spectrometry.

Liquid chromatography/mass spectrometry. The chromatographic separation of metabolites was performed using either reverse phase separation or aqueous normal phase separation online with $\mathrm{QQQ}$ mass spectrometers (Agilent Technologies, Santa Clara, CA, USA). The reverse phase chromatographic method employed a gradient containing water (solvent $\mathrm{A}$ ) and acetonitrile (solvent $B$, with both solvents containing $0.2 \%$ acetic acid and $0.1 \%$ formic acid). Separation of metabolites was performed on a Zorbax Eclipse XDB-C18 column $\left(50 \times 4.6 \mathrm{~mm}\right.$ i.d.; $1.8 \mu \mathrm{m}$, Agilent Technologies) maintained at $37^{\circ} \mathrm{C}$. The binary pump flow rate was $0.2 \mathrm{ml} / \mathrm{min}$ with a gradient spanning $2 \%$ B to $95 \%$ B over a 25 min time period. In addition, an aqueous normal phase chromatographic separation was also used for targeted identification of metabolites. This employed solvents containing acetonitrile (solvent A): water (solvent B), with both solvents modified by the addition of $0.2 \%$ acetic acid and $0.1 \%$ formic acid. The binary pump flow rate was $0.5 \mathrm{ml} / \mathrm{min}$ with a gradient spanning $95 \%$ B to $2 \%$ B over a $20 \mathrm{~min}$ period. Metabolites were separated on a Diamond Hydride column $(4 \mu \mathrm{m}$, $100 \mathrm{~A} 2.1 \times 150 \mathrm{~mm}$, MicroSolv Technology, Eatontown, NJ, USA), which was maintained in temperature-controlled chamber $\left(37^{\circ} \mathrm{C}\right)$. All the columns used in this study were washed and reconditioned after every 50 injections.

Drug treatment. WT RA and OIR-treated mice were treated with MDL 72527 in DMSO, $39 \mathrm{mg}$ per $\mathrm{kg}$ of body weight, intraperitoneal) on P7 and P8, killed on P9 and retinas were collected for further analyses. Vehicle groups received intraperitoneal injections of diluted DMSO.

Amplex red assay for $\mathrm{H}_{2} \mathrm{O}_{2}$ formation Analysis of hydrogen peroxide $\left(\mathrm{H}_{2} \mathrm{O}_{2}\right)$ formation was done using Amplex Red Hydrogen Peroxide/Peroxidase Assay Kit (Life Technologies), according to manufacturer's instructions.

Statistical analysis. One-way ANOVA with Tukey multiple comparisons or Student's t-test were used for analyzing data.

\section{Conflict of Interest}

The authors declare no conflict of interest.

Acknowledgements. This work has been supported by AHA 11SDG7440088 (SPN), VA Research Career Scientist Award (RBC), VA Merit Review Award (RBC); PHS grants EY011766 (RBC \& RWC), HL070215 (RWC) and NIH 1R01CA133458-01 (ASK).

1. Felderhoff-Mueser U, Bittigau $P$, Sifringer M, Jarosz B, Korobowicz E, Mahler L et al. Oxygen causes cell death in the developing brain. Neurobiol Dis 2004; 17: 273-282.

2. Gerstner B, Desilva TM, Genz K, Armstrong A, Brehmer F, Neve RL et al. Hyperoxia causes maturation-dependent cell death in the developing white matter. J Neurosci 2008; 28: 1236-1245.

3. Yis U, Kurul SH, Kumral A, Cilaker S, Tugyan K, Genc S et al. Hyperoxic exposure leads to cell death in the developing brain. Brain Dev 2008; 30: 556-562.
4. O'Connor AR, Stephenson T, Johnson A, Tobin MJ, Moseley MJ, Ratib S et al. Long-term ophthalmic outcome of low birth weight children with and without retinopathy of prematurity. Pediatrics 2002; 109: 12-18.

5. Barnaby AM, Hansen RM, Moskowitz A, Fulton AB. Development of scotopic visual thresholds in retinopathy of prematurity. Invest Ophthalmol Vis Sci 2007; 48: 4854-4860.

6. Fulton $A B$, Hansen RM. Photoreceptor function in infants and children with a history of mild retinopathy of prematurity. J Opt Soc Am A Opt Image Sci Vis 1996; 13: 566-571.

7. Fulton $A B$, Hansen RM, Petersen RA, Vanderveen DK. The rod photoreceptors in retinopathy of prematurity: an electroretinographic study. Arch Ophthalmol 2001; 119 : 499-505

8. Wu WC, Lin RI, Shih CP, Wang NK, Chen YP, Chao AN et al. Visual acuity, optical components, and macular abnormalities in patients with a history of retinopathy of prematurity. Ophthalmology 2012; 119: 1907-1916

9. Dembinska O, Rojas LM, Varma DR, Chemtob S, Lachapelle P. Graded contribution of retinal maturation to the development of oxygen-induced retinopathy in rats. Invest Ophthalmol Vis Sci 2001; 42: 1111-1118.

10. Liu K, Akula JD, Hansen RM, Moskowitz A, Kleinman MS, Fulton AB. Development of the electroretinographic oscillatory potentials in normal and ROP rats. Invest Ophthalmol Vis Sci 2006; 47: 5447-5452.

11. Akula JD, Mocko JA, Benador IY, Hansen RM, Favazza TL, Vyhovsky TC et al. The neurovascular relation in oxygen-induced retinopathy. Mol Vis 2008; 14: 2499-2508.

12. Akula JD, Hansen RM, Tzekov R, Favazza TL, Vyhovsky TC, Benador IY et al. Visual cycle modulation in neurovascular retinopathy. Exp Eye Res 2010; 91: 153-161.

13. Sennlaub F, Courtois $Y$, Goureau O. Inducible nitric oxide synthase mediates retinal apoptosis in ischemic proliferative retinopathy. J Neurosci 2002; 22: 3987-3993.

14. Brafman A, Mett I, Shafir M, Gottlieb H, Damari G, Gozlan-Kelner S et al. Inhibition of oxygen-induced retinopathy in RTP801-deficient mice. Invest Ophthalmol Vis Sci 2004; 45: 3796-3805.

15. Downie LE, Pianta MJ, Vingrys AJ, Wilkinson-Berka JL, Fletcher EL. Neuronal and glial cell changes are determined by retinal vascularization in retinopathy of prematurity. $J$ Comp Neurol 2007; 504: 404-417.

16. Fletcher EL, Downie LE, Hatzopoulos K, Vessey KA, Ward MM, Chow CL et al. The significance of neuronal and glial cell changes in the rat retina during oxygen-induced retinopathy. Doc Ophthalmol 2010; 120: 67-86.

17. Stevenson L, Matesanz N, Colhoun L, Edgar K, Devine A, Gardiner TA et al. Reduced nitro-oxidative stress and neural cell death suggests a protective role for microglial cells in TNFalpha-/- mice in ischemic retinopathy. Invest Ophthalmol Vis Sci 2010; 51: 3291-3299.

18. Narayanan SP, Suwanpradid J, Saul A, Xu Z, Still A, Caldwell RW et al. Arginase 2 deletion reduces neuro-glial injury and improves retinal function in a model of retinopathy of prematurity. PLoS One 2011; 6: e22460.

19. Dzietko M, Boos V, Sifringer M, Polley O, Gerstner B, Genz K et al. A critical role for Fas/ $\mathrm{CD}-95$ dependent signaling pathways in the pathogenesis of hyperoxia-induced brain injury. Ann Neurol 2008: 64: 664-673.

20. Vottier G, Pham H, Pansiot J, Biran V, Gressens P, Charriaut-Marlangue C et al. Deleterious effect of hyperoxia at birth on white matter damage in the newborn rat. Dev Neurosci 2011; 33: 261-269.

21. Natoli R, Valter K, Chrysostomou V, Stone J, Provis J. Morphological, functional and gene expression analysis of the hyperoxic mouse retina. Exp Eye Res 2011; 92: 306-314.

22. Geller S, Krowka R, Valter K, Stone J. Toxicity of hyperoxia to the retina: evidence from the mouse. Adv Exp Med Biol 2006; 572: 425-437.

23. Wellard J, Lee D, Valter K, Stone J. Photoreceptors in the rat retina are specifically vulnerable to both hypoxia and hyperoxia. Vis Neurosci 2005; 22: 501-507.

24. Dorfman AL, Chemtob S, Lachapelle P. Postnatal hyperoxia and the developing rat retina: beyond the obvious vasculopathy. Doc Ophthalmol 2010; 120: 61-66.

25. Elms SC, Toque HA, Rojas M, Xu Z, Caldwell RW, Caldwell RB. The role of arginase I in diabetes-induced retinal vascular dysfunction in mouse and rat models of diabetes. Diabetologia 2013; 56: 654-662.

26. Romero MJ, Iddings JA, Platt DH, Ali MI, Cederbaum SD, Stepp DW et al. Diabetes-induced vascular dysfunction involves arginase I. Am J Physiol Heart Circ Physiol 2012; 302: H159-H166.

27. Chen B, Calvert AE, Cui H, Nelin LD. Hypoxia promotes human pulmonary artery smooth muscle cell proliferation through induction of arginase. Am J Physiol Lung Cell Mol Physiol 2009; 297: L1151-L1159.

28. Jin Y, Calvert TJ, Chen B, Chicoine LG, Joshi M, Bauer JA et al. Mice deficient in Mkp-1 develop more severe pulmonary hypertension and greater lung protein levels of arginase in response to chronic hypoxia. Am J Physiol Heart Circ Physiol 2010; 298: H1518-H1528.

29. Hein TW, Zhang C, Wang W, Chang Cl, Thengchaisri N, Kuo L. Ischemia-reperfusion selectively impairs nitric oxide-mediated dilation in coronary arterioles: counteracting role of arginase. FASEB J 2003; 17: 2328-2330.

30. Jeyabalan G, Klune JR, Nakao A, Martik N, Wu G, Tsung A et al. Arginase blockade protects against hepatic damage in warm ischemia-reperfusion. Nitric Oxide 2008; 19 : 29-35.

31. Morrison LD, Kish SJ. Brain polyamine levels are altered in Alzheimer's disease. Neurosci Lett 1995; 197: 5-8

32. Gomes-Trolin C, Nygren I, Aquilonius SM, Askmark H. Increased red blood cell polyamines in ALS and Parkinson's disease. Exp Neurol 2002; 177: 515-520. 
33. Zahedi K, Huttinger F, Morrison R, Murray-Stewart T, Casero RA, Strauss KI. Polyamine catabolism is enhanced after traumatic brain injury. J Neurotrauma 2010; 27: 515-525.

34. Wood PL, Khan MA, Kulow SR, Mahmood SA, Moskal JR. Neurotoxicity of reactive aldehydes: the concept of 'aldehyde load' as demonstrated by neuroprotection with hydroxylamines. Brain Res 2006; 1095: 190-199.

35. Takano K, Ogura M, Nakamura Y, Yoneda Y. Neuronal and glial responses to polyamines in the ischemic brain. Curr Neurovasc Res 2005; 2: 213-223.

36. Ivanova S, Batliwalla F, Mocco J, Kiss S, Huang J, Mack W et al. Neuroprotection in cerebral ischemia by neutralization of 3-aminopropanal. Proc Natl Acad Sci USA 2002; 99: 5579-5584.

37. Pernet $\mathrm{V}$, Bourgeois $\mathrm{P}$, Di Polo $\mathrm{A}$. A role for polyamines in retinal ganglion cell excitotoxic death. J Neurochem 2007; 103: 1481-1490.

38. Seiler N. Oxidation of polyamines and brain injury. Neurochem Res 2000; 25: 471-490.

39. Kruman I, Bruce-Keller AJ, Bredesen D, Waeg G, Mattson MP. Evidence that 4-hydroxynonenal mediates oxidative stress-induced neuronal apoptosis. I Neurosci 1997; 17: 5089-5100.

40. Ivanova S, Botchkina GI, Al-Abed Y, Meistrell M III, Batliwalla F, Dubinsky JM et al. Cerebral ischemia enhances polyamine oxidation: identification of enzymatically formed 3-aminopropanal as an endogenous mediator of neuronal and glial cell death. J Exp Med 1998; 188: 327-340.

41. Ong WY, Lim HM, Lim TM, Lutz B. Kainate-induced neuronal injury leads to persistent phosphorylation of CAMP response element-binding protein in glial and endothelial cells in the hippocampus. Exp Brain Res 2000; 131: 178-186.

42. McCracken E, Valeriani V, Simpson C, Jover T, McCulloch J, Dewar D. The lipid peroxidation by-product 4-hydroxynonenal is toxic to axons and oligodendrocytes. J Cereb Blood Flow Metab 2000; 20: 1529-1536.

43. Morrow EM, Belliveau MJ, Cepko CL. Two phases of rod photoreceptor differentiation during rat retinal development. J Neurosci 1998; 18: 3738-3748.

44. Narayanan SP, Rojas M, Suwanpradid J, Toque HA, Caldwell RW, Caldwell RB. Arginase in retinopathy. Prog Retin Eye Res 2013; 36: 260-280.

45. Casero RA, Pegg AE. Polyamine catabolism and disease. Biochem J 2009; 421: 323-338.

46. Fulton AB, Hansen RM, Moskowitz A, Barnaby AM. Multifocal ERG in subjects with a history of retinopathy of prematurity. Doc Ophthalmol 2005; 111: 7-13.

47. Lachapelle P, Dembinska O, Rojas LM, Benoit J, Almazan G, Chemtob S. Persistent functional and structural retinal anomalies in newborn rats exposed to hyperoxia. Can $J$ Physiol Pharmacol 1999; 77: 48-55.

48. Morris SM Jr. Recent advances in arginine metabolism: roles and regulation of the arginases. Br J Pharmacol 2009; 157: 922-930.

49. Felderhoff-Mueser U, Taylor DL, Greenwood K, Kozma M, Stibenz D, Joashi UC et al. Fas/CD95/APO-1 can function as a death receptor for neuronal cells in vitro and in vivo and is upregulated following cerebral hypoxic-ischemic injury to the developing rat brain. Brain Pathol 2000; 10: 17-29.
50. Graham EM, Sheldon RA, Flock DL, Ferriero DM, Martin LJ, O'Riordan DP et al. Neonatal mice lacking functional Fas death receptors are resistant to hypoxic-ischemic brain injury. Neurobiol Dis 2004; 17: 89-98.

51. Sifringer M, Bendix I, Borner C, Endesfelder S, von Haefen C, Kalb A et al. Prevention of neonatal oxygen-induced brain damage by reduction of intrinsic apoptosis. Cell Death Dis 2012; 3: e250.

52. Luo X, Budihardjo I, Zou H, Slaughter C, Bid Wang X. A Bcl2 interacting protein, mediates cytochrome $c$ release from mitochondria in response to activation of cell surface death receptors. Cell 1998; 94: 481-490.

53. Clarke P, Beckham JD, Leser JS, Hoyt CC, Tyler KL. Fas-mediated apoptotic signaling in the mouse brain following reovirus infection. J Virol 2009; 83: 6161-6170.

54. Mudduluru M, Zubrow AB, Ashraf QM, Delivoria-Papadopoulos M, Mishra OP. Tyrosine phosphorylation of apoptotic proteins during hyperoxia in mitochondria of the cerebral cortex of newborn piglets. Neurochem Res 2010; 35: 1003-1009.

55. Pocernich CB, Butterfield DA. Acrolein inhibits NADH-linked mitochondrial enzyme activity: implications for Alzheimer's disease. Neurotox Res 2003; 5: 515-520.

56. Yu Z, Li W, Hillman J, Brunk UT. Human neuroblastoma (SH-SY5Y) cells are highly sensitive to the lysosomotropic aldehyde 3-aminopropanal. Brain Res 2004; 1016: 163-169.

57. Yu Z, Li W, Brunk UT. 3-Aminopropanal is a lysosomotropic aldehyde that causes oxidative stress and apoptosis by rupturing lysosomes. Apmis 2003; 111: 643-652.

58. Basu HS, Thompson TA, Church DR, Clower CC, Mehraein-Ghomi F, Amlong CA et al. A small molecule polyamine oxidase inhibitor blocks androgen-induced oxidative stress and delays prostate cancer progression in the transgenic adenocarcinoma of the mouse prostate model. Cancer Res 2009; 69: 7689-7695.

59. Dogan A, Rao AM, Hatcher J, Rao VL, Baskaya MK, Dempsey RJ. Effects of MDL 72527, a specific inhibitor of polyamine oxidase, on brain edema, ischemic injury volume, and tissue polyamine levels in rats after temporary middle cerebral artery occlusion. $J$ Neurochem 1999; 72: 765-770.

60. Shi O, Morris Jr SM, Zoghbi H, Porter CW, O'Brien WE. Generation of a mouse model for arginase II deficiency by targeted disruption of the arginase II gene. Mol Cell Biol 2001; 21: 811-813.

(c) $(1)(\theta)$ Cell Death and Disease is an open-access journal cor licensed under a Creative Commons Attribution-NonCommercialNoDerivs 3.0 Unported License. To view a copy of this license, visit http://creativecommons.org/licenses/by-nc-nd/3.0/

Supplementary Information accompanies this paper on Cell Death and Disease website (http://www.nature.com/cddis) 